\title{
Correction to: Therapeutic novelties in migraine: new drugs, new hope?
}

Thien Phu Do, Song Guo and Messoud Ashina*

Correction to: J Headache Pain (2019) 20:37

https://doi.org/10.1186/s10194-019-0974-3

After publication of the original article [1], the authors have notified us that the row corresponding to the "Alniditan" drug shouldn't have been included in Table 1. Table 1 should therefore be presented as below:

Table 1 Overview of ditans in alphabetical order

\begin{tabular}{ll}
\hline Drug & Status \\
\hline Lasmiditan (COL-144) & Phase III clinical trials \\
LY-334370 & Development terminated
\end{tabular}

Received: 9 May 2019 Accepted: 9 May 2019

Published online: 17 May 2019

\section{Reference}

1. Do TP et al (2019) Therapeutic novelties in migraine: new drugs, new hope? J Headache Pain 20:37. https://doi.org/10.1186/s10194-019-0974-3

\footnotetext{
*Correspondence: ashina@dadlnet.dk
}

The original article can be found online at https://doi.org/10.1186/s10194019-0974-3

Danish Headache Center and Department of Neurology, Rigshospitalet Glostrup, Faculty of Health Sciences, University of Copenhagen, Copenhagen, Denmark 